\title{
Modern Mathematical Physics: what it should be
}

\author{
L. D. Faddeev
}

February 4, 2008

When somebody asks me, what I do in science, I call myself a specialist in mathematical physics. As I have been there for more than 40 years, I have some definite interpretation of this combination of words: "mathematical physics." Cynics or purists can insist that this is neither mathematics nor physics, adding comments with a different degree of malice. Naturally, this calls for an answer, and in this short essay I want to explain briefly my understanding of the subject. It can be considered as my contribution to the discussion about the origin and role of mathematical physics and thus to be relevant for this volume.

The matter is complicated by the fact that the term "mathematical physics" (often abbreviated by MP in what follows) is used in different senses and can have rather different content. This content changes with time, place and person.

I did not study properly the history of science; however, it is my impression that, in the beginning of the twentieth century, the term MP was practically equivalent to the concept of theoretical physics. Not only Henri Poincaré, but also Albert Einstein, were called mathematical physicists. Newly established theoretical chairs were called chairs of mathematical physics. It follows from the documents in the archives of the Nobel Committee that MP had a right to appear both in the nominations and discussion of the candidates for the Nobel Prize in physics [1]. Roughly speaking, the concept of MP covered theoretical papers where mathematical formulae were used.

However, during an unprecedented bloom of theoretical physics in the 20s and 30s, an essential separation of the terms "theoretical" and "mathematical" occurred. For many people, MP was reduced to the important but auxiliary course "Methods of Mathematical Physics" including a set of useful mathematical tools. The monograph of P. Morse and H. Feshbach [2] is a classical example of such a course, addressed to a wide circle of physicists and engineers.

On the other hand, MP in the mathematical interpretation appeared as a theory of partial differential equations and variational calculus. The monographs of R. Courant and D. Hilbert [3] and S. Sobolev [- 4 ] are outstanding illustrations of this development. The theorems of existence and uniqueness based on the variational principles, a priori estimates, and imbedding theorems for functional spaces comprise the main content of this direction. As a student of O. Ladyzhenskaya, I was immersed in this subject since the 3rd year of my 
undergraduate studies at the Physics Department of Leningrad University. My fellow student N. Uraltseva now holds the chair of MP exactly in this sense.

MP in this context has as its source mainly geometry and such parts of classical mechanics as hydrodynamics and elasticity theory. Since the 60 s a new impetus to MP in this sense was supplied by Quantum Theory. Here the main apparatus is functional analysis, including the spectral theory of operators in Hilbert space, the mathematical theory of scattering and the theory of Lie groups and their representations. The main subject is the Schrödinger operator. Though the methods and concrete content of this part of MP are essentially different from those of its classical counterpart, the methodological attitude is the same. One sees the quest for the rigorous mathematical theorems about results which are understood by physicists in their own way.

I was born as a scientist exactly in this environment. I graduated from the unique chair of Mathematical Physics, established by V.I. Smirnov at the Physics Department of Leningrad University already in the 30s. In his venture V.I. Smirnov got support from V. Fock, the world famous theoretical physicist with very wide mathematical interests. Originally this chair played the auxiliary role of being responsible for the mathematical courses for physics students. However in 1955 it got permission to supervise its own diploma projects, and I belonged to the very first group of students using this opportunity. As I already mentioned, O.A. Ladyzhenskaya was our main professor. Although her own interests were mostly in nonlinear PDE and hydrodynamics, she decided to direct me to quantum theory. During last two years of undergraduate studies I was to read the monograph of K.O. Friedrichs, "Mathematical Aspects of Quantum Field Theory," and relate it to our group of 5 students and our professor on a special seminar. At the same time my student friends from the chair of Theoretical Physics were absorbed in reading the first monograph on Quantum Electrodynamics by A. Ahieser and V. Berestevsky. The difference in attitudes and language was striking and I was to become accustomed to both.

After my graduation O.A. Ladyzhenskaya remained my tutor but she left me free to choose research topics and literature to read. I read both mathematical papers (i.e. on direct and inverse scattering problems by I.M. Gelfand and B.M. Levitan, V.A. Marchenko, M.G. Krein, A.Ya. Povzner) and "Physical Review" (i.e. on formal scattering theory by M. Gell-Mann, M. Goldberger, J. Schwinger and H. Ekstein) as well. Papers by I. Segal, L. Van-Hove and R. Haag added to my first impressions on Quantum Field Theory taken from K. Friederichs. In the process of this self-education my own understanding of the nature and goals of MP gradually deviated from the prevailing views of the members of the V. Smirnov chair. I decided that it is more challenging to do something which is not known to my colleagues from theoretical physics rather than supply theorems of substantiality. My first work on the inverse scattering problem especially for the many-dimensional Schrödinger operator and that on the three body scattering problem confirm that I really tried to follow this line of thought.

This attitude became even firmer when I began to work on Quantum Field Theory in the middle of the $60 \mathrm{~s}$. As a result, my understanding of the goal of MP 
drastically modified. I consider as the main goal of MP the use of mathematical intuition for the derivation of really new results in the fundamental physics. In this sense, MP and Theoretical Physics are competitors. Their goals in unraveling the laws of the structure of matter coincide. However, the methods and even the estimates of the importance of the results of work may differ quite significally.

Here it is time to say in what sense I use the term "fundamental physics." The adjective "fundamental" has many possible interpretations when applied to the classification of science. In a wider sense it is used to characterize the research directed to unraveling new properties of physical systems. In the narrow sense it is kept only for the search for the basic laws that govern and explain these properties.

Thus, all chemical properties can be derived from the Schrödinger equation for a system of electrons and nuclei. Alternatively, we can say that the fundamental laws of chemistry in a narrow sense are already known. This, of course, does not deprive chemistry of the right to be called a fundamental science in a wide sense.

The same can be said about classical mechanics and the quantum physics of condensed matter. Whereas the largest part of physical research lies now in the latter, it is clear that all its successes including the theory of superconductivity and superfluidity, Bose-Einstein condensation and quantum Hall effect have a fundamental explanation in the nonrelativistic quantum theory of many body systems.

An unfinished physical fundamental problem in a narrow sense is physics of elementary particles. This puts this part of physics into a special position. And it is here where modern MP has the most probable chances for a breakthrough.

Indeed, until recent time, all physics developed along the traditional circle: experiment - theoretical interpretation - new experiment. So the theory traditionally followed the experiment. This imposes a severe censorship on the theoretical work. Any idea, bright as it is, which is not supplied by the experimental knowledge at the time when it appeared is to be considered wrong and as such must be abandoned. Characteristically the role of censors might be played by theoreticians themselves and the great L. Landau and W. Pauli were, as far as I can judge, the most severe ones. And, of course, they had very good reason.

On the other hand, the development of mathematics, which is also to a great extent influenced by applications, has nevertheless its internal logic. Ideas are judged not by their relevance but more by esthetic criteria. The totalitarianism of theoretical physics gives way to a kind of democracy in mathematics and its inherent intuition. And exactly this freedom could be found useful for particle physics. This part of physics traditionally is based on the progress of accelerator techniques. The very high cost and restricted possibilities of the latter soon will become an uncircumventable obstacle to further development. And it is here that mathematical intuition could give an adequate alternative. This was already stressed by famous theoreticians with mathematical inclinations. Indeed, let me cite a paper [5] by P. Dirac from the early 30s: 
The steady progress of physics requires for its theoretical formulation a mathematics that gets continually more advanced. This is only natural and to be expected. What, however, was not expected by the scientific workers of the last century was the particular form that the line of advancement of the mathematics would take, namely, it was expected that the mathematics would get more complicated, but would rest on a permanent basis of axioms and definitions, while actually the modern physical developments have required a mathematics that continually shifts its foundations and gets more abstract. Non-euclidean geometry and non-commutative algebra, which were at one time considered to be purely fictions of the mind and pastimes for logical thinkers, have now been found to be very necessary for the description of general facts of the physical world. It seems likely that this process of increasing abstraction will continue in the future and that advance in physics is to be associated with a continual modification and generalization of the axioms at the base of mathematics rather than with logical development of any one mathematical scheme on a fixed foundation.

There are at present fundamental problems in theoretical physics awaiting solution, e.g., the relativistic formulation of quantum mechanics and the nature of atomic nuclei (to be followed by more difficult ones such as the problem of life), the solution of which problems will presumably require a more drastic revision of our fundamental concepts than any that have gone before. Quite likely these changes will be so great that it will be beyond the power of human intelligence to get the necessary new ideas by direct attempts to formulate the experimental data in mathematical terms. The theoretical worker in the future will therefore have to proceed in a more inderect way. The most powerful method of advance that can be suggested at present is to employ all the resources of pure mathematics in attempts to perfect and generalise the mathematical formalism that forms the existing basis of theoretical physics, and after each success in this direction, to try to interpret the new mathematical features in terms of physical entities.

Similar views were expressed by C.N. Yang. I did not find a compact citation, but all spirit of his commentaries to his own collection of papers [6] shows this attitude. Also he used to tell this to me in private discussions.

I believe that the dramatic history of setting the gauge fields as a basic tool in the description of interactions in Quantum Field Theory gives a good illustration of the influence of mathematical intuition on the development of the fundamental physics. Gauge fields, or Yang-Mills fields, were introduced to the wide audience of physicists in 1954 in a short paper by C.N. Yang and R. Mills [7], dedicated to the generalization of the electromagnetic fields and the corresponding principle of gauge invariance. The geometric sense of this principle for the electromagnetic field was made clear as early as in the late 20 s due to the 
papers of V. Fock [8] and H. Weyl [9]. They underlined the analogy of the gauge (or gradient in the terminology of V. Fock) invariance of the electrodynamics and the equivalence principle of the Einstein theory of gravitation. The gauge group in electrodynamics is commutative and corresponds to the multiplication of the complex field (or wave function) of the electrically charged particle by a phase factor depending on the space-time coordinates. Einstein's theory of gravity provides an example of a much more sophisticated gauge group, namely the group of general coordinate transformation. Both H. Weyl and V. Fock were to use the language of the moving frame with spin connection, associated with local Lorentz rotations. Thus the Lorentz group became the first nonabelian gauge group and one can see in [8] essentially all formulas characteristics of nonabelian gauge fields. However, in contradistinction to the electromagnetic field, the spin connection enters the description of the space-time and not the internal space of electric charge.

In the middle of the 30s, after the discovery of the isotopic spin in nuclear physics, and forming the Yukawa idea of the intermediate boson, O. Klein tried to geometrise these objects. His proposal was based on his 5-dimensional picture. Proton and neutron (as well as electron and neutrino, there were no clear distinction between strong and weak interactions) were put together in an isovector and electromagnetic field and charged vector meson comprised a $2 \times 2$ matrix. However the noncommutative $S U(2)$ gauge group was not mentioned.

Klein's proposal was not received favorably and N. Borh did not recommend him to publish a paper. So the idea remained only in the form of contribution to proceedings of Warsaw Conference "New Theories in Physics" [10].

The noncommutative group, acting in the internal space of charges, appeared for the first time in the paper [7] of C.N. Yang and R. Mills in 1954. There is no wonder that Yang received a cool reaction when he presented his work at Princeton in 1954. The dramatic account of this event can be found in his commentaries 6. Pauli was in the audience and immediately raised the question about mass. Indeed the gauge invariance forbids the introduction of mass to the vector charged fields and masslessness leads to the long range interaction, which contradicts the experiment. The only known massless particles (and accompaning long range interactions) are photon and graviton. It is evident from Yang's text, that Pauli was well acquainted with the differential geometry of nonabelian vector fields but his own censorship did not allow him to speak about them. As we know now, the boldness of Yang and his esthetic feeling finally were vindicated. And it can be rightly said, that C.N. Yang proceeded according to mathematical intuition.

In 1954 the paper of Yang and Mills did not move to the forefront of high energy theoretical physics. However, the idea of the charged space with noncommutative symmetry group acquired more and more popularity due to the increasing number of elementary particles and the search for the universal scheme of their classification. And at that time the decisive role in the promotion of the Yang-Mills fields was also played by mathematical intuition.

At the beginning of the $60 \mathrm{~s}, \mathrm{R}$. Feynman worked on the extension of his own scheme of quantization of the electromagnetic field to the gravitation theory of 
Einstein. A purely technical difficulty — the abundance of the tensor indices made his work rather slow. Following the advice of M. Gell-Mann, he exercised first on the simpler case of the Yang-Mills fields. To his surprise, he found that a naive generalization of his diagrammatic rules designed for electrodynamics did not work for the Yang-Mills field. The unitarity of the $S$-matrix was broken. Feynman restored the unitarity in one loop by reconstructing the full scattering amplitude from its imaginary part and found that the result can be interpreted as a subtraction of the contribution of some fictitious particle. However his technique became quite cumbersome beyond one loop. His approach was gradually developed by B. De-Witt [11. It must be stressed that the physical senselessness of the Yang-Mills field did not preclude Feynman from using it for mathematical construction.

The work of Feynman 12 became one of the starting points for my work in Quantum Field Theory, which I began in the middle of the 60s together with Victor Popov. Another point as important was the mathematical monograph by A. Lichnerowitz [13], dedicated to the theory of connections in vector bundles. From Lichnerowitz's book it followed clearly that the Yang-Mills field has a definite geometric interpretation: it defines a connection in the vector bundle, the base being the space-time and the fiber the linear space of the representation of the compact group of charges. Thus, the Yang-Mills field finds its natural place among the fields of geometrical origin between the electromagnetic field (which is its particular example for the one-dimensional charge) and Einstein's gravitation field, which deals with the tangent bundle of the Riemannian spacetime manifold.

It became clear to me that such a possibility cannot be missed and, notwithstanding the unsolved problem of zero mass, one must actively tackle the problem of the correct quantization of the Yang-Mills field.

The geometric origin of the Yang-Mills field gave a natural way to resolve the difficulties with the diagrammatic rules. The formulation of the quantum theory in terms of Feynman's functional integral happened to be most appropriate from the technical point of view. Indeed, to take into account the gauge equivalence principle one has to integrate over the classes of gauge equivalent fields rather than over every individual configuration. As soon as this idea is understood, the technical realization is rather straightforward. As a result V. Popov and I came out at the end of 1966 with a set of rules valid for all orders of perturbation theory. The fictitious particles appeared as auxiliary variables giving the integral representation for the nontrivial determinant entering the measure over the set of gauge orbits.

Correct diagrammatic rules of quantization of the Yang-Mills field, obtained by V. Popov and me in 1966-1967 [14, 15], did not attract immediate the attention of physicists. Moreover, the time when our work was done was not favorable for it. Quantum Field Theory was virtually forbidden, especially in the Soviet Union, due to the influence of Landau. "The Hamiltonian is dead" - this phrase from his paper [16], dedicated to the anniversary of W. Pauli - shows the extreme of Landau's attitude. The reason was quite solid, it was based not on experiment, but on the investigation of the effects of renormal- 
ization, which led Landau and his coworkers to believe that the renormalized physical coupling constant is inevitably zero for all possible local interactions. So there was no way for Victor Popov and me to publish an extended article in a major Soviet journal. We opted for the short communication in "Physics Letters" and were happy to be able to publish the full version in the preprint series of newly opened Kiev Institute of Theoretical Physics. This preprint was finally translated into English by B. Lee as a Fermilab preprint in 1972, and from the preface to the translation it follows that it was known in the West already in 1968.

A decisive role in the successful promotion of our diagrammatic rules into physics was played by the works of G. 't Hooft [17], dedicated to the YangMills field interacting with the Higgs field (and which ultimately led to a Nobel Prize for him in 1999) and the discovery of dimensional transmutation (the term of S. Coleman [18]). The problem of mass was solved in the first case via the spontaneous symmetry breaking. The second development was based on asymptotic freedom. There exists a vast literature dedicated to the history of this dramatic development. I refer to the recent papers of G. 't Hooft [19] and D. Gross [20], where the participants in this story share their impressions of this progress. As a result, the Standard Model of unified interactions got its main technical tool. From the middle of the 70s until our time it remains the fundamental base of high energy physics. For our discourse it is important to stress once again that the paper 14 based on mathematical intuition preceded the works made in the traditions of theoretical physics.

The Standard Model did not complete the development of fundamental physics in spite of its unexpected and astonishing experimental success. The gravitational interactions, whose geometrical interpretation is slightly different from that of the Yang-Mills theory, is not included in the Standard Model. The unification of quantum principles, Lorentz-Einstein relativity and Einstein gravity has not yet been accomplished. We have every reason to conjecture that the modern MP and its mode of working will play the decisive role in the quest for such a unification.

Indeed, the new generation of theoreticians in high energy physics have received an incomparably higher mathematical education. They are not subject to the pressure of old authorities maintaining the purity of physical thinking and/or terminology. Futhermore, many professional mathematicians, tempted by the beauty of the methods used by physicists, moved to the position of the modern mathematical physics. Let use cite from the manifesto, written by P. MacPherson during the organization of the Quantum Field Theory year at the School of Mathematics of the Institute for Advanced Study at Princeton:

The goal is to create and convey an understanding, in terms congenial to mathematicians, of some fundamental notions of physics, such as quantum field theory. The emphasis will be on developing the intuition stemming from functional integrals.

One way to define the goals of the program is by negation, excluding certain important subjects commonly pursued by mathe- 
maticians whose work is motivated by physics. In this spirit, it is not planned to treat except peripherally the magnificient new applications of field theory, such as Seiberg-Witten equations to Donaldson theory. Nor is the plan to consider fundamental new constructions within mathimatics that were inspired by physics, such as quantum groups or vertex operator algebras. Nor is the aim to discuss how to provide mathematical rigor for physical theories. Rather, the goal is to develop the sort of intuition common among physicists for those who are used to thought processes stemming from geometry and algebra.

I propose to call the intuition to which MacPherson refers that of mathematical physics. I also recommend the reader to look at the instructive drawing by P. Dijkgraaf on the dust cover of the volumes of lectures given at the School [21].

The union of these two groups constitutes an enormous intellectual force.

In the next century we will learn if this force is capable of substituting for the traditional experimental base of the development of fundamental physics and pertinent physical intuition.

\section{References}

[1] B. Nagel, The Discussion Concerning the Nobel Prize of Max Planck, Science Technology and Society in the Time of Alfred Nobel (New York: Pergamon, 1982).

[2] P. Morse and H. Feshbach, Methods of Theoretical Physics, (New York: McGraw-Hill, 1953).

[3] R. Courant and D. Hilbert, Methoden der mathematischen Physik, (Berlin: Springer, 1931).

[4] S.L. Sobolev, Nekotorye primeneniya funktsional'nogo analiza v matematicheskoi fizike (Some Applications of Functional Analysis in Mathematical Physics), (Leningrad: Lenigrad. Gos. Univ., 1950).

[5] P. Dirac, Quantized Singularities in the Electromagnetic Field, Proc. Roy. Soc. London A 133, 60-72 (1931).

[6] C.N. Yang, Selected Papers 1945-1980 with Commentary, (San Francisco: Freeman, 1983).

[7] C.N. Yang and R. Mills, Conservation of Isotopic Spin and Isotopic Gauge Invariance, Phys. Rev. 96, 191-195i (1954).

[8] V. Fock, L'equation d'onde de Dirac et la geometrie de Riemann, J. Phys. et Rad. 70 392-405 (1929).

[9] H. Weyl, Electron and Gravitation, Zeit. Phys., 56, 330-352 (1929). 
[10] O. Klein, On the Theory of Charged Fields: Submitted to the Conference New Theories in Physics, Warsaw, 1938, Surv. High Energy Phys., 1986, 5 269 (1986).

[11] B. De-Witt, Quantum Theory of Gravity II: The manifest covariant theory, Phys. Rev., 1967, 162, 1195-1239 (1967).

[12] R.P. Feynman, Quantum Theory of Gravitation, Acta Phys. Polon. 24, 697-722 (1963).

[13] A. Lichnerowicz, Théorie globale des connexions et des groupes d'holonomie, (Roma: Ed. Cremonese, 1955).

[14] L. Faddeev and V. Popov, Feynman Diagrams for the Yang-Mills Field, Phys. Lett. B, 25, 29-30 (1967).

[15] V. Popov and L. Faddeev, Perturbation Theory for Gauge-Invariant Fields, Preprint, National Accelerator Laboratory, NAL-THY-57 (1972).

[16] L. Landau, in Theoretical Physics in the twentieth century, a memorial volume to Wolfgang Pauli, ed. M. Fierz and V. Weisskopf, (Cambridge, USA, 1956).

[17] G. 't Hooft, Renormalizable Lagrangians for Massive Yang-Mills Fields, Nucl. Phys. B, 35, 167-188 (1971).

[18] S. Coleman, Secret Symmetries: An Introduction to Spontaneous Symmetry Breakdown and Gauge Fields: Lecture given at 1973 International Summer School in Physics Ettore Majorana, Erice (Sicily), 1973, Erice SubnuclPhys., 1973.

[19] G. 't Hooft, When was Asumptotic Freedom Discovered? Rehabilitation of Quantum Field Theory, Preprint, hep-th/9808154 (1998).

[20] D. Gross, Twenty Years of Asymptotic Freedom, Preprint, hep-th/9809080 (1998).

[21] V. Dijkgraaf, Quantum Fields and Strings: A course for mathematicians, vols. I, II (AMS, IAS, 1999). 